\title{
Osteosarcoma with metastasis at initial diagnosis: Current outcomes and prognostic factors in the context of a comprehensive cancer center
}

\author{
SAMER SALAH ${ }^{1}$, RAMI AHMAD ${ }^{1}$, IYAD SULTAN ${ }^{2}$, \\ SAMEER YASER $^{1}$ and AHMAD SHEHADEH ${ }^{3}$ \\ Departments of ${ }^{1}$ Medical Oncology and ${ }^{2}$ Pediatric Oncology; ${ }^{3}$ Orthopedic Oncology Unit, \\ Department of Surgery, King Hussein Cancer Center, Amman 11941, Jordan
}

Received March 10, 2014; Accepted March 17, 2014

DOI: $10.3892 / \mathrm{mco} .2014 .325$

\begin{abstract}
The aim of this study was to report the outcomes and prognostic factors for a cohort of patients with osteosarcoma who presented with metastasis at initial diagnosis. Data of consecutive patients with osteosarcoma and metastatic disease at initial presentation, who were treated and followed up at a single institution, were retrospectively reviewed. The effect of potential factors on overall survival (OS) was analyzed through univariate and multivariate analysis. Between January, 2000 and March, 2013, a total of 135 patients with osteosarcoma, of whom $21(16.0 \%)$ had distant metastasis at initial presentation, were diagnosed and treated at our center and were included in this analysis. The patients were treated with a strategy that integrates multi-agent chemotherapy and resection of all sites of gross disease whenever feasible. The 5-year OS and event-free survival (EFS) were $23.0 \%$ and $11.0 \%$, respectively. The factors associated with inferior OS in the univariate analysis included the osteoblastic variant, extrapulmonary metastasis and failure to achieve complete remission. In the multivariate analysis, the osteoblastic variant [hazard ratio $(\mathrm{HR})=4.83,95 \%$ confidence interval $(\mathrm{CI}): 1.16-20.0, \mathrm{P}=0.038]$ and extrapulmonary metastasis $(\mathrm{HR}=5.0,95 \%$ CI: $1.40-17.94$, $\mathrm{P}=0.018$ ) were the only independent prognostic factors. The current outcomes of patients with osteosarcoma and metastasis at initial diagnosis remained poor. The osteoblastic subtype and extrapulmonary metastatic sites predicted poor survival in our series. To the best of our knowledge, this is the first report of the correlation between the histological subtype and survival for patients with metastatic disease at initial presentation; therefore, confirmation in future studies is required.
\end{abstract}

Correspondenceto:DrSamerSalah,MedicalOncologyDepartment, King Hussein Cancer Center, Queen Rania Al Abdullah Street, Al-Jubeiha, Amman 11941, Jordan

E-mail: ssalah@khcc.jo

Key words: osteosarcoma, metastasis, prognostic factors, overall survival, chemotherapy

\section{Introduction}

Osteosarcoma is the most common primary malignant bone tumor in children and adults $(1,2)$. Although the survival of patients with primary localized disease has improved significantly since the 1970 s with the introduction of active multi-agent chemotherapy (3-5), the outcomes of patients who present with metastatic disease remains poor.

Approximately $15 \%$ of patients with osteosarcoma present with metastatic disease at initial diagnosis (6). A number of studies investigated the effect of potential prognostic factors on survival; however, those series were mostly limited to pediatric populations (6-9), which makes any interpretation of the data irrelevant for the adult population. Furthermore, the majority of the previous studies included patients who were treated in an older era, with less consistent follow-up schedules and imaging modalities.

In the present study, we report the outcomes and analyze the prognostic factors of adult and pediatric patients with osteosarcomas who presented with metastatic disease at initial diagnosis and who were treated at a single institution in the context of the current era of the multimodality therapy.

\section{Materials and methods}

Screening for eligible patients. We retrospectively reviewed the charts of patients with osteosarcoma of the extremities who had metastasis at initial presentation and who were treated and followed up at the King Hussein Cancer Center between January, 2000 and March, 2013.

The following data were retrieved from the medical records of the patients following acquisition of Institutional Review Board approval: Patient age at diagnosis; gender; histological osteosarcoma subtype; sites of metastasis at the time of initial diagnosis; serum alkaline phosphatase (ALP) level at diagnosis; status of the primary tumor (controlled or uncontrolled); therapeutic modalities, including details of any pulmonary metastasectomy (PM) and surgical therapy of the primary tumor; details of systemic chemotherapy, including date of initiation of first-line chemotherapy; and dates of the last follow-up or death. 
Patients with skip metastasis within the same bone by the primary tumor were excluded if they did not have other sites of distant metastasis.

Staging at diagnosis. All the patients with pathologically confirmed diagnosis of osteosarcoma at our center underwent magnetic resonance imaging (MRI) of the primary tumor, computed tomography (CT) scan of the chest and bone scan. Patients with suspicious bone scan findings underwent additional imaging studies to confirm the presence of bone metastasis.

Follow-up with imaging studies. The follow-up protocols and imaging schedules were consistent at our center during the eligibility period. Follow-up with CT scan of the chest and MRI of the extremities corresponding to the location of the primary tumor was typically performed after every 2 cycles of chemotherapy. Following completion of chemotherapy, imaging studies were performed at 3-month intervals or at any reporting of symptoms that were suggestive of disease progression (DP).

All the radiological assessments were interpreted in accordance to the response evaluation criteria in solid tumors as follows: Complete remission (CR), disappearance of all metastatic lesions; partial remission (PR), $\geq 30 \%$ decrease in the sum of the diameters of the target lesions; DP, appearance of $\geq 1$ new lesions or an increase in the size of target measurable lesions of $\geq 20 \%$ of the sum of the longest diameters; and stable disease (SD), neither sufficient shrinkage to qualify for PR nor sufficient increase to qualify for DP.

Therapeutic strategies. All the patients were assessed for surgical resection of the metastasis and the primary tumor whenever feasible. This surgical approach is typically staged and is integrated with perioperative chemotherapy, which includes the same chemotherapeutic protocols that are utilized for patients with localized primary disease. Resectability is defined as the ability to surgically remove the metastasis and the primary tumor without postoperative residual disease.

The patients were treated according to two first-line chemotherapy protocols. Adult patients were treated with cisplatin $(100 \mathrm{mg} / \mathrm{m} 2)$ and doxorubicin $(75 \mathrm{mg} / \mathrm{m} 2)$, both administered on day 1 every 21 days and continued for up to 6 cycles, DP or limiting toxicity. Pediatric patients received high-dose methotrexate (MTX) $12 \mathrm{~g} / \mathrm{m}^{2}$ integrated into their primary cisplatin and doxorubicin regimen. Resection of the metastasis and the primary tumor were typically attempted after 3-4 cycles if the disease was resectable and there was no evidence of DP following chemotherapy.

Statistical analysis. Survival curves were calculated according to the Kaplan-Meier method and were compared by means of the log-rank test. $\mathrm{P}<0.05$ was considered to indicate a statistically significant difference.

Overall survival (OS) was calculated from the time of diagnosis until the last follow-up or death. Event-free survival (EFS) was calculated from the time of diagnosis until the first documentation of progression or recurrence, last follow-up or death. Factors that were identified as significant according to the univariate analysis, were entered into the multivariate
Table I. Demographics and disease-related characteristics for the 21 patients with metastatic osteosarcoma at presentation.

\begin{tabular}{|c|c|}
\hline Clinical variables & $\begin{array}{c}\text { Patient no. }(\%) \\
\quad(n=21)\end{array}$ \\
\hline \multicolumn{2}{|l|}{ Age, years } \\
\hline$<18$ & $10(48.0)$ \\
\hline$\geq 18$ & $11(52.0)$ \\
\hline \multicolumn{2}{|l|}{ Gender } \\
\hline Male & $12(57.0)$ \\
\hline Female & $9(43.0)$ \\
\hline \multicolumn{2}{|l|}{ Primary tumor location } \\
\hline Femur & $11(52.0)$ \\
\hline Tibia & $8(38.0)$ \\
\hline Humerous & $2(10.0)$ \\
\hline \multicolumn{2}{|l|}{ Histological subtypes } \\
\hline Osteoblastic & $6(29.0)$ \\
\hline Osteosarcoma-NOS & $8(38.0)$ \\
\hline Other & $7(33.0)$ \\
\hline \multicolumn{2}{|l|}{ Treatment of primary tumor } \\
\hline LSS & $11(52.0)$ \\
\hline Amputation & $4(19.0)$ \\
\hline No surgery & $6(29.0)$ \\
\hline \multicolumn{2}{|l|}{ Metastatic sites } \\
\hline Lung & $15(72.0)$ \\
\hline Lung and extrapulmonary & $3(14.0)$ \\
\hline Extrapulmonary & $3(14.0)$ \\
\hline \multicolumn{2}{|l|}{ Treatment } \\
\hline PM and chemotherapy & $4(19.0)$ \\
\hline Chemotherapy alone & $15(71.0)$ \\
\hline BSC alone & $2(10.0)$ \\
\hline
\end{tabular}

NOS, not otherwise specified; LSS, limb salvage surgery; PM, pulmonary metastasectomy; BSC, best supportive care.

analysis utilizing the backward stepwise Cox regression model. All the statistical analyses were performed with SPSS software, version 20 (SPSS, Inc., Chicago, IL, USA).

\section{Results}

Eligible patients. Between January, 2000 and March, 2013, a total of 135 patients with osteosarcoma of the extremity were treated and followed up at our institution. Of these patients, 25 had metastatic disease at presentation. Four patients were excluded for skip metastasis in the same bone involved by the primary tumor, without distant metastasis in other sites, leaving 21 patients eligible for this analysis. All the patients had pathological confirmation of their diagnosis at our center. The characteristics of the 21 patients are summarized in Table I.

The median patient age at diagnosis was 18 years (range, 5-32 years). The median interval from the first symptoms until referral to our center was 7 months (range, 3-13 months). The 
Table II. Results of univariate analysis for event-free survival (EFS) and overall survival (OS) outcomes of the 21 patients with metastatic osteosarcoma at presentation.

\begin{tabular}{|c|c|c|c|c|c|}
\hline Variables & $\begin{array}{l}\text { Patient no. }(\%) \\
\quad(\mathrm{n}=21)\end{array}$ & $\begin{array}{l}\text { Median EFS, } \\
\text { months }\end{array}$ & $\begin{array}{l}\text { Log-rank } \\
\text { P-value }\end{array}$ & $\begin{array}{l}\text { Median OS, } \\
\text { months }\end{array}$ & $\begin{array}{c}\text { Log-rank } \\
\text { P-value }\end{array}$ \\
\hline Age, years & & & 0.089 & & 0.48 \\
\hline$<18$ & $10(48.0)$ & 4.0 & & 17.5 & \\
\hline$\geq 18$ & $11(52.0)$ & 9.0 & & 32.9 & \\
\hline Gender & & & 0.83 & & 0.75 \\
\hline Male & $12(57.0)$ & 5.0 & & 19.6 & \\
\hline Female & $9(43.0)$ & 7.7 & & 16.3 & \\
\hline Histological subtype & & & 0.84 & & 0.040 \\
\hline Osteoblastic & $6(29.0)$ & 5.4 & & 10.5 & \\
\hline Other & $15(71.0)$ & 4.0 & & 32.9 & \\
\hline Alkaline phosphatase, IU/l & & & 0.11 & & 0.26 \\
\hline$<500$ & $18(86.0)$ & 7.2 & & 32.9 & \\
\hline$\geq 500$ & $3(14.0)$ & 5.0 & & 17.0 & \\
\hline Primary tumor site & & & 0.53 & & 0.57 \\
\hline Femur & $10(48.0)$ & 7.2 & & 19.6 & \\
\hline Other & $11(52.0)$ & 4.0 & & 32.9 & \\
\hline Status of primary tumor & & & 0.31 & & 0.32 \\
\hline Controlled & $15(71.0)$ & 5.4 & & 19.6 & \\
\hline Uncontrolled & $6(29.0)$ & 5.0 & & 17.7 & \\
\hline Necrosis following CTX & & & 0.099 & & 0.21 \\
\hline$\geq 90 \%$ & $3(14.0)$ & 35.0 & & 42.0 & \\
\hline Other & $18(86.0)$ & 5.4 & & 17.5 & \\
\hline Achievement of CR & & & 0.026 & & 0.046 \\
\hline Yes & $4(19.0)$ & 35 & & 42.0 & \\
\hline No & $17(81.0)$ & 5.0 & & 17.5 & \\
\hline First-line chemotherapy & & & 0.48 & & 0.61 \\
\hline Cisplatin + doxorubicin & $11(52.0)$ & 7.2 & & 32.9 & \\
\hline Cisplatin, doxorubicin + MTX & $7(33.0)$ & 3.7 & & 19.6 & \\
\hline IE chemotherapy & $1(5.0)$ & & & & \\
\hline No chemotherapy & $2(10.0)$ & & & & \\
\hline Site of metastasis & & & 0.35 & & 0.014 \\
\hline Lung only & $15(71.0)$ & 5.0 & & 33.6 & \\
\hline Extrapulmonary & $6(29.0)$ & 5.4 & & 10.5 & \\
\hline Bone metastasis & & & 0.22 & & 0.07 \\
\hline Yes & $5(24.0)$ & 5.4 & & 17 & \\
\hline No & $16(76.0)$ & 7.2 & & 33.6 & \\
\hline Number of metastatic sites & & & 0.44 & & 0.06 \\
\hline One & $16(76.0)$ & 5.4 & & 33.6 & \\
\hline More than one & $5(24.0)$ & 6.4 & & 17 & \\
\hline
\end{tabular}

CTX, chemotherapy; CR, complete remission; MTX, methotrexate; IE, ifosfamide and etoposide.

patients had a median follow-up time of 17.5 months (range, 6.4-78 months).

The most common metastatic site was the lungs (18 patients, $86.0 \%$ ). Of the 18 patients with lung metastasis, 3 had concomitant extrapulmonary metastasis, involving the bone in 2 patients and the liver and cardiac muscle in the third patient. In addition, 3 patients presented with extrapulmonary metastasis without lung metastasis ( 2 with bone and bone marrow metastasis and 1 with bone metastasis alone). Overall, 5 patients $(24.0 \%)$ had bone metastasis at initial presentation.

During the course of metastatic disease, the lungs remained the most common metastatic site, affecting 20 patients 
(95.0\%), whereas bone metastasis affected 7 (33.0\%), bone marrow metastasis $2(10.0 \%)$, liver metastasis $2(10.0 \%)$ and brain metastasis 1 patient $(5.0 \%)$ (data not shown).

Therapeutic modalities. Eleven patients (52.0\%) underwent resection of their primary tumor with limb salvage surgery (LSS) and $4(19.0 \%)$ underwent an amputation, whereas $6(29.0 \%)$ did not undergo resection of their primary tumor ( 3 of the patients refused amputation) (Table I). The metastasis was deemed resectable for 7 patients (33.0\%); however, only 4 patients underwent PM in addition to resection of the primary tumor, whereas the remaining 3 were not treated with PM, since they refused amputation and, as such, their metastases were not amenable to resection with curative intent due to the presence of uncontrolled primary tumor.

The main reasons for the metastasis being deemed unresectable in 14 patients were the presence of numerous bilateral lung metastases in $6(43.0 \%)$, metastasis involving $\geq 1$ organ in $5(36.0 \%)$ and refusal of amputation in 3 patients $(21.0 \%)$.

Overall, 4 patients (19.0\%) achieved complete remission (CR) (Table II), all following PM and resection of the primary tumor in addition to multi-agent chemotherapy.

A total of 19 patients $(90.0 \%)$ were treated with chemotherapy. The first-line chemotherapy included cisplatin and doxorubicin in 11 patients, cisplatin, doxorubicin and MTX in 7 patients and ifosfamide and etoposide in 1 patient. The remaining 2 patients $(10.0 \%)$ were treated with best supportive care only for poor performance status (Table II).

Survival outcome. At the time of analysis, 13 patients $(62.0 \%)$ had succumbed to their disease and 2 remained alive without evidence of disease for $>5$ years from their initial diagnosis (at 70 and 78 months following PM).

The 5-year OS and EFS for the entire cohort were 23.0 and $11.0 \%$, respectively. The univariate analysis identified 3 factors that were correlated with inferior OS, namely the osteoblastic subtype of the tumor, failure to achieve CR and presence of extrapulmonary metastases. Failure to achieve CR was the only factor that was found to be correlated with inferior EFS in the univariate analysis (Table II).

The multivariate analysis identified the osteoblastic subtype and the presence of extrapulmonary metastasis as the only independent predictors for inferior OS (Table III).

The survival of patients with these two adverse factors was extremely poor. Patients with the osteoblastic subtype had a median OS of 10.5 months, whereas patients with the other subtypes had a median OS of 32.9 months (Fig. 1). Likewise, the presence of extrapulmonary metastasis predicted inferior OS compared to that of patients with metastases limited to the lungs (10.5 vs. 33.6 months, respectively; Fig. 2). Patients with any of these two independent prognostic factors were at $\sim 5$-fold increased mortality risk.

Finally, we compared the OS outcomes for 3 groups of patients based on the number of independent prognostic factors present; patients exhibiting both these independent risk factors $(n=3)$ had a median OS of only 6.4 months, patients with 1 risk factor $(n=7)$ had a median OS of 17.0 months and patients with none of the independent adverse prognostic factors $(n=11)$ had a median OS of 33.6 months, $\mathrm{P}=0.001$ (data not shown).
Table III. Results of multivariate analysis for factors affecting overall survival.

\begin{tabular}{lccc}
\hline Variables & Hazard ratio & $95 \%$ CI & P-value \\
\hline $\begin{array}{l}\text { Histological subtype } \\
\text { Osteoblastic variant }\end{array}$ & 4.83 & $1.16-20.0$ & 0.038 \\
Other variants & 1 & & \\
Pattern of metastasis & & $1.40-17.94$ & 0.018 \\
Lung only & 1 & & \\
Other patterns & 5.0 & & \\
Achievement of CR & & $0.46-17.37$ & 0.24 \\
Yes & 1 & & \\
No & 2.84 & & \\
\hline
\end{tabular}

CI, confidence interval; CR, complete remission.

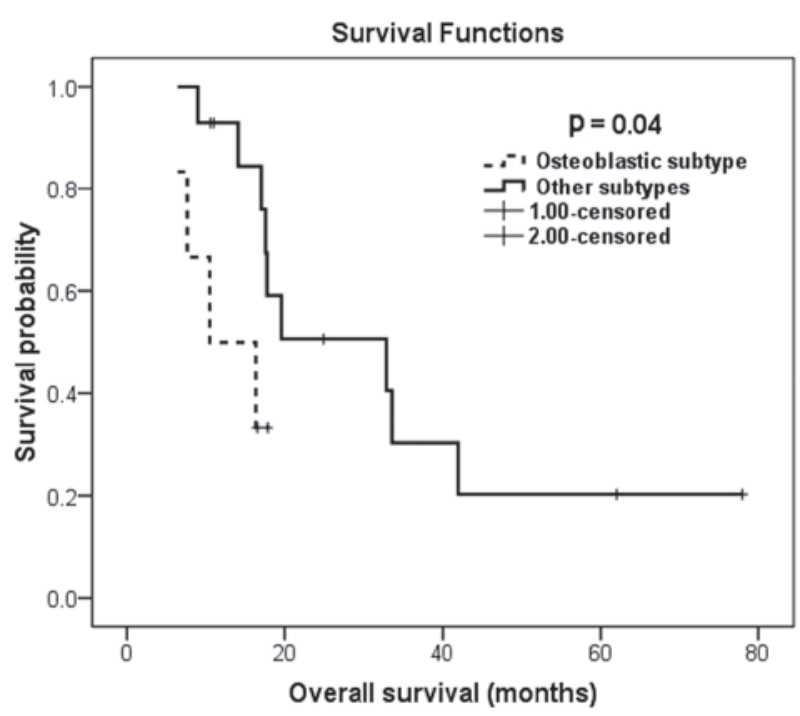

Figure 1. Kaplan-Meier overall survival estimation according to the histological subtype.

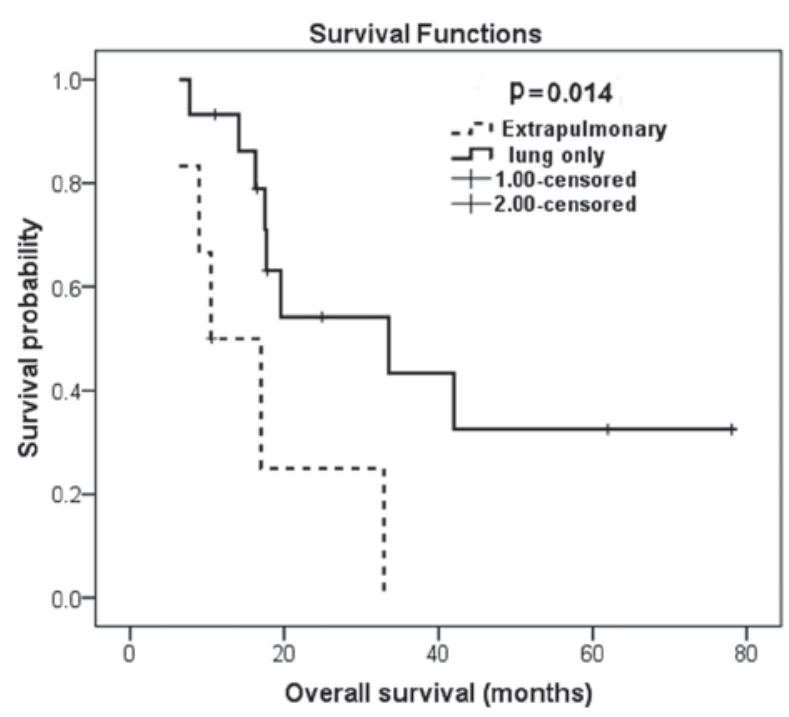

Figure 2. Kaplan-Meier overall survival estimation according to the pattern of metastatic disease at presentation. 
Efficacy of chemotherapy. Of the 19 patients who were treated with chemotherapy, 6 (32.0\%) achieved an objective response, $8(42.0 \%)$ had SD and the remaining patients had refractory primary tumors and exhibited DP. The median progression-free survival (PFS) for all 19 patients was 5.4 [95\% confidence interval (CI): 2.7-8.1 months]. Only 1 patient (17.0\%) with the osteoblastic subtype achieved an objective response compared to $5(33.0 \%)$ of patients with the other subtypes [P = not statistically significant (NS)].

We did not observe a significant difference in PFS between patients who had MTX incorporated into their primary cisplatin and doxorubicin regimen and those who did not (median PFS, 3.8 vs. 5.5 months, respectively, $\mathrm{P}=0.38$ ). Furthermore, the 5-year OS did not differ between the two regimens (26 vs. $29 \%$ respectively, $\mathrm{P}=0.61$ ).

\section{Discussion}

The present study clearly demonstrates that patients with metastatic osteosarcoma at initial presentation are heterogeneous and include different subgroups with significant differences in prognosis.

Patients with osteosarcoma and resectable metastases at initial presentation should be managed in a way that is similar to patients with no metastatic disease, i.e., with a surgical approach aimed at complete resection of all the lesions and multi-agent chemotherapy, as there is a chance for cure when treated with such an approach; however, patients with multi-organ metastasis who are beyond resectability should be managed with a palliative intent.

In our series, the achievement of CR following surgery was the only factor that correlated with improvement regarding EFS as well as OS. This finding indicates that achievement of complete surgical remission is the most important predictor for cure. Consistent with our data, completeness of resection predicted a favorable OS for patients with metastatic osteosarcoma at diagnosis in almost all the studies available in the literature (9-12). Achievement of CR following complete surgical resection is also the most consistent predictor of survival in the relapse setting (13-16).

In our cohort, a notable finding was the low proportion of patients who had undergone complete resection of the metastasis (19.0\%), which was significantly lower compared to the proportion of patients who had undergone resection of the metastasis in other series $(9,11)$; a likely explanation for this discrepancy is that our patients presented late, at a more advanced stage of metastatic disease, with a higher proportion of patients with numerous and bilateral lung metastases compared to the patients included in previous studies $(6,9,10)$. This more advanced stage is likely due to late referrals, which lead to a delay in diagnosis and initiation of therapy, as the median time from first reporting the symptoms until presentation to our center was 7 months, which is significantly higher compared to the 2.8-month interval from symptoms to diagnosis reported by Mialou et al (9). This finding highlights the importance of early referral, as delays may result in presenting with metastatic disease that is beyond resectability.

Consistent with our findings, patients with combined metastatic sites fared significantly worse ccompared to patients with metastasis limited to the lungs (11). However, the potential effect of the histological subtype on patient outcomes was not commonly considered, as only a limited number of studies addressed it in the analysis of potential prognostic factors $(10,17)$. Although the osteoblastic subtype was an independent predictor of inferior OS in our cohort, Daw et al (10) did not identify a significant correlation between osteoblastic subtype and patient survival.

To the best of our knowledge, our study is the first to suggest that the osteoblastic subtype may exert an independent effect on survival of patients with metastatic osteosarcoma at diagnosis. Bearing in mind that our series was limited by the small sample size, this finding must be interpreted with caution and confirmation from additional studies is required.

Our study did not confirm an association between the degree of pathological necrosis following preoperative chemotherapy and survival, although such a correlation was previously reported (11); similarly, we did not identify any prognostic value for high serum ALP, in contrast to what was reported by Mialou et al (9). We believe that the small number of patients was responsible for the differences based on those factors not reaching statistical significance in our study.

We acknowledge that the small sample size, which renders exploratory sub-analyses not feasible, is an important limitation. An additional limitation is that we were unable to assess the quality of life for the patients included in this analysis, due to the retrospective design. However, our data is relevant to pediatric as well as adult patients, as half of our patient population were adults, a population for which data availability in the literature regarding metastatic osteosarcoma is quite limited. In addition, our data represent the current outcomes of treating metastatic osteosarcoma at diagnosis, as all the included patients were treated after the year 2000, unlike other series that reported the outcomes of patients who were treated in an era of less advanced supportive care and less consistent imaging modalities and schedules.

In conclusion, complete resection of the metastases in addition to multi-agent chemotherapy is the cornerstone of management of patients with metastatic osteosarcoma at initial diagnosis. The presence of extrapulmonary metastasis and osteoblastic tumor subtype predicted inferior survival for our series; however, further confirmation of the effect of histological subtype on survival is required.

\section{References}

1. Dorfman HD and Czerniak B: Bone cancers. Cancer 75 (Suppl 1): 203-210, 1995.

2. Nagarajan R, Weigel BJ, Thompson RC and Perentesis JP: Osteosarcoma in the first decade of life. Med Pediatr Oncol 41: 480-483, 2003.

3. Friedman MA and Carter SK: The therapy of osteogenic sarcoma: current status and thoughts for the future. J Surg Oncol 4: 482-510, 1972.

4. Link MP, Goorin AM, Miser AW, et al: The effect of adjuvant chemotherapy on relapse-free survival in patients with osteosarcoma of the extremity. N Engl J Med 314: 1600-1606, 1986.

5. Link MP, Goorin AM, Horowitz M, et al: Adjuvant chemotherapy of high-grade osteosarcoma of the extremity. Clin Orthop Relat Res 270: 8-14, 1991

6. Kaste SC, Pratt CB, Cain AM, Jones-Wallace DJ and Rao BN: Metastases detected at the time of diagnosis of primary pediatric extremity osteosarcoma at diagnosis: imaging features. Cancer 86: 1602-1608, 1999. 
7. Marina NM, Pratt CB, Rao BN, Shema SJ and Meyer WH: Improved prognosis of children with osteosarcoma metastatic to the lung(s) at the time of diagnosis. Cancer 70: 2722-2727, 1999.

8. Ferguson WS, Harris MB, Goorin AM, et al: Presurgical window of carboplatin and surgery and multidrug chemotherapy for the treatment of newly diagnosed metastatic or unresectable osteosarcoma: Pediatric Oncology Group Trial. J Pediatr Hematol Oncol 23: 340-348, 2001.

9. Mialou V, Philip T, Kalifa C, et al: Metastatic osteosarcoma at diagnosis: prognostic factors and long-term outcome - the French pediatric experience. Cancer 104: 1100-1109, 2005.

10. Daw NC, Billups CA, Rodriguez-Galindo C, et al: Metastatic osteosarcoma. Cancer 106: 403-412, 2006.

11. Kager L, Zoubek A, Potschger U, et al: Primary metastatic osteosarcoma: presentation and outcome of patients treated on neoadjuvant Cooperative Osteosarcoma Study Group protocols. J Clin Oncol 21: 2011-2018, 2003.

12. Meyers PA, Heller G, Healey JH, et al: Osteogenic sarcoma with clinically detectable metastasis at initial presentation. J Clin Oncol 11: 449-453, 1993.
13. Ferrari S, Briccoli A, Mercuri M, et al: Postrelapse survival in osteosarcoma of the extremities: prognostic factors for long-term survival. J Clin Oncol 21: 710-715, 2003.

14. Pastorino U, Gasparini M, Tavecchio L, et al: The contribution of salvage surgery to the management of childhood osteosarcoma. J Clin Oncol 9: 1357-1362, 1991.

15. Leary SE, Wozniak AW, Billups CA, et al: Survival of pediatric patients after relapsed osteosarcoma: the St. Jude Children's Research Hospital experience. Cancer 119: 2645-2653, 2013.

16. Chou AJ, Merola PR, Wexler LH, et al: Treatment of osteosarcoma at first recurrence after contemporary therapy: the Memorial Sloan-Kettering Cancer Center experience. Cancer 104: 2214-2221, 2005.

17. Buddingh EP, Anninga JK, Versteegh MI, et al: Prognostic factors in pulmonary metastasized high-grade osteosarcoma. Pediatr Blood Cancer 54: 216-221, 2010. 